Kragujevac Journal of Mathematics

Volume 44(2) (2020), Pages 165-179.

\title{
ON THE ESTRADA INDEX OF POINT ATTACHING STRICT $k$-QUASI TREE GRAPHS
}

\author{
MOHAMMAD A. IRANMANESH ${ }^{1}$ AND RAZIYEH NEJATI ${ }^{2}$
}

\begin{abstract}
Let $G=(V, E)$ be a finite and simple graph with $\lambda_{1}, \lambda_{2}, \ldots, \lambda_{n}$ as its eigenvalues. The Estrada index of $G$ is $E E(G)=\sum_{i=1}^{n} e^{\lambda_{i}}$. For a positive integer $k$, a connected graph $G$ is called strict $k$-quasi tree if there exists a set $U$ of vertices of size $k$ such that $G \backslash U$ is a tree and this is as small as possible with this property. In this paper, we define point attaching strict $k$-quasi tree graphs and obtain the graph with minimum Estrada index among point attaching strict $k$-quasi tree graphs with $k$ even cycles.
\end{abstract}

\section{INTRODUCTION}

Let $G=(V(G), E(G))$ be a finite and simple graph of order $n$, where by $V(G)$ and $E(G)$ we denote the set of vertices and edges, respectively. Let $A(G)$ be the adjacency matrix of $G$, and $\lambda_{1}, \lambda_{2}, \ldots, \lambda_{n}$ be its eigenvalues. The Estrada index of $G$ is defined as

$$
E E(G)=\sum_{i=1}^{n} e^{\lambda_{i}}
$$

which was first proposed by Estrada in 2000 [6]. We refer reader to $[7,8,15,16]$ for multiple applications of Estrada index in various fields, for example in network science and biochemistry. The results for trees can be found in $[3,10,13,19]$. Gutman approximated the Estrada index of cycles and paths in [9]. The unicyclic graphs with maximum and minimum Estrada index have been determined in [5]. Recently, the Esrada index of the cactus graphs in which every block is a triangle, has been characterized in $[11,12]$.

A connected graph $G$ is called quasi tree if there exists $v_{0} \in V(G)$ such that $G \backslash\left\{v_{0}\right\}$ is a tree. Lu in [14] has determined the Randić index of quasi trees. The Harary index

Key words and phrases. Estrada Index, quasi tree graph, point attaching Strict $k$-quasi tree graph. 2010 Mathematics Subject Classification. Primary: 05C35. Secondary: 05C50.

DOI 10.46793/KgJMat2002.165I

Received: February 20, 2018.

Accepted: March 14, 2018. 
of quasi tree graphs and generalized quasi tree graphs are presented in [18]. A strict $k$-quasi tree graph $G$ is a connected graph which is not a tree, and $k$ is the smallest positive integer such that there exists a $k$-element subset $U$ of vertices for which $G \backslash U$ is a tree.

Let $G$ be a connected graph constructed from pairwise disjoint connected graphs $G_{1}, G_{2}, \ldots, G_{d}$ as follows: select a vertex of $G_{1}$, a vertex of $G_{2}$, and identify these two vertices. Then continue in this manner inductively. More precisely, suppose that we have already used $G_{1}, G_{2}, \ldots, G_{i}$ in the construction, where $2 \leq i \leq d-1$. Then select a vertex in the already constructed graph (which may in particular be one of the already selected vertices) and a vertex $G_{i+1}$; and identify these two vertices. Note that the graph $G$ constructed in this way has a tree-like structure, the $G_{i}$ 's being its building stones. We will briefly say that $G$ is obtained by point attaching from $G_{1}, G_{2}, \ldots, G_{d}$ and that $G_{i}$ 's are the primary subgraphs of $G$ [4].

A graph $G$ is said to be point attaching strict $k$-quasi, if it is constructed from primary subgraphs $G_{1}, G_{2}, \ldots, G_{d}$ where each primary subgraph $G_{i}$ is a strict $k_{i}$-quasi tree graph for each $1 \leq i \leq d$, and $k=\sum_{i=1}^{d} k_{i}$.

In this paper we study the Estrada index of point attaching strict $k$-quasi graphs.

\section{PRELIMINARIES}

For $\ell \in \mathbb{N} \cup\{0\}$, let $S_{\ell}(G)=\sum_{i=1}^{n} \lambda_{i}^{\ell}$ be the $\ell^{\text {th }}$ spectral moment of $G$, which is equal to the number of closed walks of length $\ell$ in $G$ [2]. For every graph $G$, we have $S_{0}(G)=n, S_{1}(G)=\mathbf{C}, S_{2}(G)=2 m, S_{3}(G)=6 \mathbf{D}$, and $S_{4}(G)=2 \sum_{i=1}^{n} d_{i}^{2}-2 m+8 \mathbf{Q}$, where $n, \mathbf{C}, m, \mathbf{D}, \mathbf{Q}$ denote the number of vertices, the number of loops, the number of edges, the number of triangles and the number of quadrangles in $G$, respectively and $d_{i}=d_{i}(G)$ is the degree of vertex $v_{i}$ in $G$ [2]. Bearing in mind the Taylor expansion of $e^{x}$, we have the following equation for the Estrada index of graph $G$,

$$
E E(G)=\sum_{i=1}^{n} e^{\lambda_{i}}=\sum_{i=1}^{n} \sum_{\ell=0}^{\infty} \frac{\lambda_{i}^{\ell}}{\ell !}=\sum_{\ell=0}^{\infty} \frac{S_{\ell}(G)}{\ell !} .
$$

It follows from Equation 2.1 that $E E(G)$ is a strictly monotonously increasing function of $S_{\ell}(G)$. Let $G_{1}$ and $G_{2}$ be two graphs. If $S_{\ell}\left(G_{1}\right) \leq S_{\ell}\left(G_{2}\right)$ holds for all positive integer $\ell$, then $E E\left(G_{1}\right) \leq E E\left(G_{2}\right)$. Moreover, if the strict inequality $S_{\ell}\left(G_{1}\right)<S_{\ell}\left(G_{2}\right)$ holds for at least one value $\ell_{0} \geq 0$, then $E E\left(G_{1}\right)<E E\left(G_{2}\right)$.

Recall that a sequence $a_{0}, a_{1}, \ldots, a_{n}$ of numbers is said to be unimodal if for some $0 \leq i \leq n$ we have $a_{0} \leq a_{1} \leq \cdots \leq a_{i} \geq a_{i+1} \geq \cdots \geq a_{n}$, and this sequence is called symmetric if $a_{i}=a_{n-i}$ for $0 \leq i \leq n$ [17]. Thus a symmetric unimodal sequence $a_{0}, a_{1}, \ldots, a_{n}$ has its maximum at the middle term ( $n$ even) or middle two terms $(n$ odd). Let $A$ be the adjacency matrix of the graph $G$. It is well-known that the entry $\left(A^{\ell}\right)_{i, j}$ represents the number of walks of length $\ell$ from vertex $v_{i}$ to vertex $v_{j}$ [1]. Obviously, $\left(A^{\ell}\right)_{i, j}=\left(A^{\ell}\right)_{j, i}$ for undirected graphs.

Throughout this paper, $\Gamma(k)$ is a point attaching strict $k$-quasi tree graph with $k$ even cycles (see Figure 1) and $M_{\ell}(G)$ denotes the set of closed walks of length $\ell$ in $G$, 
and we show that among all point attaching strict $k$-quasi tree graphs with $k$ even cycles, $\Gamma(k)$ is the graph with minimum Estrada index.

\section{The Number of Closed Walks of Length $\ell$ in $\Gamma(k)$}

Let $M_{\ell}(k(c-1), i)$ denote the set of closed walks of length $\ell$ starting at the vertex $v_{i}$ in $\Gamma(k)$ with $k$ even cycles of length $c$ and $\left|M_{\ell}(k(c-1), i)\right|=S_{\ell}(k(c-1), i)$ denote the number of closed walks of length $\ell$ starting at the vertex $v_{i}$ in $\Gamma(k)$ (see Figure 1 ).

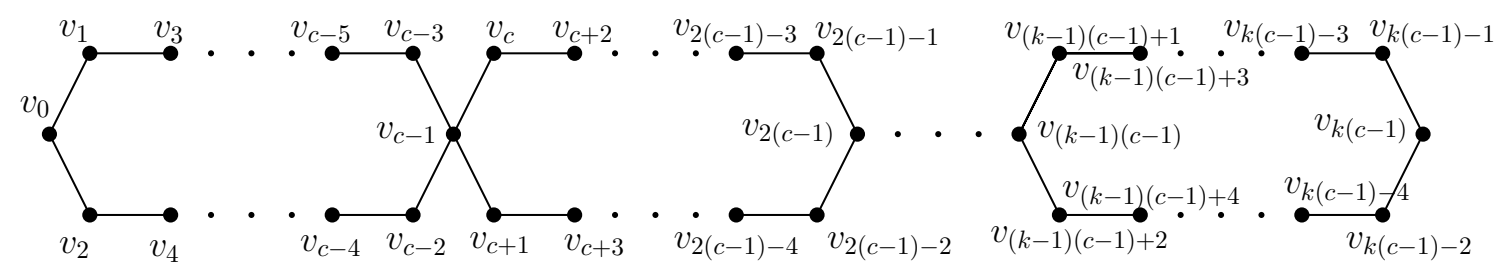

Figure 1. The graph $\Gamma(k)$.

Lemma 3.1. The map $\varphi: V(\Gamma(k)) \longrightarrow V(\Gamma(k))$, given by $\varphi\left(v_{i}\right)=v_{k(c-1)-i}$ is an automorphism.

Proof. One can easily see that $\varphi$ is bijective. Let vertices $v_{i}$ and $v_{j}$ be adjacent. Then by definition of $\varphi$, we have the following cases.

(i) $\varphi\left(v_{0}\right)=v_{k(c-1)}$ and $\varphi\left(v_{k(c-1)}\right)=v_{0}$.

(ii) $i=t(c-1), 0<t<k$. In this case $v_{j} \in\left\{v_{i-1}, v_{i-2}, v_{i+1}, v_{i+2}\right\}$. Hence, $k(c-1)-i=k(c-1)-t(c-1)=(k-t)(c-1)$. This implies that $\varphi\left(v_{i}\right)=v_{(k-t)(c-1)}$.

We will only prove the case $v_{j}=v_{i-1}$. A similar argument can be used for other cases. If $v_{j}=v_{i-1}$, then $k(c-1)-j=k(c-1)-t(c-1)+1=$ $(k-t)(c-1)+1$. Hence $\varphi\left(v_{j}\right)=v_{(k-t)(c-1)+1}$ which is adjacent to $\varphi\left(v_{i}\right)$.

(iii) $i=t(c-1)+s, 0<t \leqslant k-1,1 \leqslant s \leqslant c-2$. In this case $v_{j} \in\left\{v_{i-2}, v_{i+2}\right\}$. Hence, $k(c-1)-j=k(c-1)-t(c-1)-s=(k-t)(c-1)-s$. This implies that $\varphi\left(v_{i}\right)=v_{(k-t)(c-1)-s}$.

If $v_{j}=v_{i-2}$, then $k(c-1)-t(c-1)-s+2=(k-t)(c-1)-s+2$. Hence, $\varphi\left(v_{j}\right)=v_{(k-t)(c-1)-s+2}$ which is adjacent to $\varphi\left(v_{i}\right)$. The proof for case $v_{j}=v_{i+2}$ is similar.

Corollary 3.1. Let $A$ be the adjacency matrix of the point attaching strict $k$-quasi tree graph $\Gamma(k)$. Then $\left(A^{\ell}\right)_{i, j}=\left(A^{\ell}\right)_{k(c-1)-i, k(c-1)-j}$ for $0 \leqslant i, j \leqslant k(c-1)$.

Proof. This is an immediate consequence of Lemma 3.1. 
Lemma 3.2. If $k \geqslant 2$ and $t$ are integers and $0 \leqslant t \leqslant c-2$, then:

$$
\begin{aligned}
S_{\ell}(k(c-1), t) & \leq S_{\ell}(k(c-1), t+(c-1)) \\
& \leq \cdots \leq S_{\ell}\left(k(c-1), t+\left(\left[\frac{k}{2}\right]-1\right)(c-1)\right) \\
& \leq S_{\ell}\left(k(c-1), t+\left[\frac{k}{2}\right](c-1)\right),
\end{aligned}
$$

where $\ell \geq c-1$. If $\ell \geq\left[\frac{k}{2}\right]$, then strict inequalities hold.

Proof. We prove every diagonal and the main diagonal of the matrix $A^{\ell}$ are unimodal. By Lemma 3.1, $\left(A^{\ell}\right)_{t, j}=\left(A^{\ell}\right)_{k(c-1)-t, k(c-1)-j}$. So we only need to show that the diagonals paralleling to the main diagonal increase for $t+j \leqslant k(c-1)$.

By induction on integer $\ell$, we will show that for every $j \leqslant k(c-1)$ where $t+j+$ $2 c-2 \leqslant k(c-1)$, we have:

$$
\left(A^{\ell}\right)_{t+c-1, j+c-1} \geqslant\left(A^{\ell}\right)_{t, j}
$$

By the definition of $\Gamma(k)$ we have $A_{t, j}=1$ if and only if $A_{t+c-1, j+c-1}=1$. Therefore, the result is hold for $\ell=1$. Assume that the result holds for integer $\ell$. There are four cases as follows.

Case 1: $t, j \equiv 0(\bmod (c-1))$.

Since the set of walks of length $\ell+1$ from $v_{t}$ to $v_{j}$ is in bijective correspondence with the set of walks of length $\ell$ from $v_{t}$ to $v_{h}$ adjacent to $v_{j}$, so

$$
\begin{aligned}
\left(A^{\ell+1}\right)_{t+c-1, j+c-1}= & \left(A^{\ell}\right)_{t+c-1, j+c-2}+\left(A^{\ell}\right)_{t+c-1, j+c-3}+\left(A^{\ell}\right)_{t+c-1, j+c} \\
& +\left(A^{\ell}\right)_{t+c-1, j+c+1}, \\
\left(A^{\ell+1}\right)_{t, j}= & \left(A^{\ell}\right)_{t, j-1}+\left(A^{\ell}\right)_{t, j-2}+\left(A^{\ell}\right)_{t, j+1}+\left(A^{\ell}\right)_{t, j+2} .
\end{aligned}
$$

By the induction hypothesis, we have the following results:

$$
\begin{aligned}
\left(A^{\ell}\right)_{t+c-1, j+c-2} & \geq\left(A^{\ell}\right)_{t, j-1}, \\
\left(A^{\ell}\right)_{t+c-1, j+c} & \geq\left(A^{\ell}\right)_{t, j+1}, \quad \text { for } t+j+2 \leq k(c-1), \\
\left(A^{\ell}\right)_{t+c-1, j+c-3} & \geq\left(A^{\ell}\right)_{t, j-2}, \\
\left(A^{\ell}\right)_{t+c-1, j+c+1} & \geq\left(A^{\ell}\right)_{t, j+2}, \quad \text { for } t+j+2 \leq k(c-1) .
\end{aligned}
$$

Hence, we have $\left(A^{\ell+1}\right)_{t+c-1, j+c-1} \geq\left(A^{\ell+1}\right)_{t, j}$. In addition we will show that for $\ell \geq[k(c-1) / 2]$ the strict inequalities hold.

For the strict inequality, let $1 \leq r \leq k$ be a fixed number, we consider two rows $r(c-1)$ and $(r-1)(c-1), j \leq k(c-1)$. Then

$$
\left(A^{\ell+1}\right)_{r(c-1), c-1}=\left(A^{\ell}\right)_{r(c-1), c-2}+\left(A^{\ell}\right)_{r(c-1), c-3}+\left(A^{\ell}\right)_{r(c-1), c}+\left(A^{\ell}\right)_{r(c-1), c+1}
$$

and

$$
\left(A^{\ell+1}\right)_{(r-1)(c-1), 0}=\left(A^{\ell}\right)_{(r-1)(c-1), 1}+\left(A^{\ell}\right)_{(r-1)(c-1), 2} .
$$


Note that, since $\Gamma(k)$ is symmetric we have,

$$
\begin{aligned}
\left(A^{\ell}\right)_{r(c-1), c-2} & =\left(A^{\ell}\right)_{r(c-1), c-3}>0, \\
\left(A^{\ell}\right)_{r(c-1), c} & =\left(A^{\ell}\right)_{r(c-1), c+1}>0, \\
\left(A^{\ell}\right)_{r(c-1), 1} & =\left(A^{\ell}\right)_{r(c-1), 2}>0,
\end{aligned}
$$

for $\ell \geq r(c-1)$. So,

$$
\left(A^{\ell+1}\right)_{r(c-1), c-1}=2\left(A^{\ell}\right)_{r(c-1), c-2}+2\left(A^{\ell}\right)_{r(c-1), c+1}
$$

and

$$
\left(A^{\ell+1}\right)_{(r-1)(c-1), 0}=2\left(A^{\ell}\right)_{(r-1)(c-1), 2} .
$$

By the induction hypothesis, the following inequality holds:

$$
\left(A^{\ell}\right)_{r(c-1), c+1} \geq\left(A^{\ell}\right)_{(r-1)(c-1), 2} .
$$

Thus, we have the strict inequality $\left(A^{\ell+1}\right)_{r(c-1), c-1}>\left(A^{\ell+1}\right)_{(r-1)(c-1), 0}$. This causes the chain of strict inequalities

$$
\begin{aligned}
& \left(A^{\ell+2}\right)_{r(c-1), 2(c-1)}>\left(A^{\ell+2}\right)_{(r-1)(c-1), c-1}, \\
& \left(A^{\ell+3}\right)_{r(c-1), 3(c-1)}>\left(A^{\ell+3}\right)_{(r-1)(c-1), 2(c-1)} .
\end{aligned}
$$

Finally, we have

$$
\left(A^{\ell+(k-r+1)}\right)_{r(c-1),(k-r+1)(c-1)}>\left(A^{\ell+(k-r+1)}\right)_{(r-1)(c-1),(k-r)(c-1)} .
$$

Case $2: t \equiv 0(\bmod (c-1))$ and $j \not \equiv 0(\bmod (c-1))$. Let $j \equiv 1(\bmod (c-1))$. Then

$$
\begin{aligned}
\left(A^{\ell+1}\right)_{t+c-1, j+c-1} & =\left(A^{\ell}\right)_{t+c-1, j+c-2}+\left(A^{\ell}\right)_{t+c-1, j+c+1}, \\
\left(A^{\ell+1}\right)_{t, j} & =\left(A^{\ell}\right)_{t, j-1}+\left(A^{\ell}\right)_{t, j+2} .
\end{aligned}
$$

Similarly, by the induction hypothesis, we have

$$
\begin{aligned}
& \left(A^{\ell}\right)_{t+c-1, j+c-2} \geq\left(A^{\ell}\right)_{t, j-1}, \\
& \left(A^{\ell}\right)_{t+c-1, j+c+1} \geq\left(A^{\ell}\right)_{t, j+2}, \quad \text { for } t+j+2 \leq k(c-1) .
\end{aligned}
$$

Hence, we have $\left(A^{\ell+1}\right)_{t+c-1, j+c-1} \geq\left(A^{\ell+1}\right)_{t, j}$.

In addition for the strict inequality, let $1 \leq r \leq k$ be a fixed number, we consider two rows $r(c-1)$ and $(r-1)(c-1)$. Then

$$
\begin{aligned}
\left(A^{\ell+1}\right)_{r(c-1), c}= & \left(A^{\ell}\right)_{r(c-1), c-1}+\left(A^{\ell}\right)_{r(c-1), c+2}=\left(A^{\ell-1}\right)_{r(c-1), c-2} \\
& +\left(A^{\ell-1}\right)_{r(c-1), c-3}+\left(A^{\ell-1}\right)_{r(c-1), c}+\left(A^{\ell-1}\right)_{r(c-1), c+1}+\left(A^{\ell}\right)_{r(c-1), c+2}
\end{aligned}
$$

and

$$
\begin{aligned}
\left(A^{\ell+1}\right)_{(r-1)(c-1), 1} & =\left(A^{\ell}\right)_{(r-1)(c-1), 0}+\left(A^{\ell}\right)_{(r-1)(c-1), 3} \\
& =\left(A^{\ell-1}\right)_{(r-1)(c-1), 1}+\left(A^{\ell-1}\right)_{(r-1)(c-1), 2}+\left(A^{\ell}\right)_{(r-1)(c-1), 3} .
\end{aligned}
$$


Note that, since $\Gamma(k)$ is symmetric we have,

$$
\begin{gathered}
\left(A^{\ell-1}\right)_{r(c-1), c-2}=\left(A^{\ell-1}\right)_{r(c-1), c-3}>0 \\
\left(A^{\ell-1}\right)_{r(c-1), c}=\left(A^{\ell-1}\right)_{r(c-1), c+1}>0 \\
\left(A^{\ell-1}\right)_{r(c-1), 1}=\left(A^{\ell-1}\right)_{r(c-1), 2}>0
\end{gathered}
$$

for $\ell \geq r(c-1)$.

So,

$$
\left(A^{\ell+1}\right)_{r(c-1), c}=2\left(A^{\ell-1}\right)_{r(c-1), c-2}+2\left(A^{\ell-1}\right)_{r(c-1), c}+\left(A^{\ell}\right)_{r(c-1), c+2}
$$

and

$$
\left(A^{\ell+1}\right)_{(r-1)(c-1), 1}=2\left(A^{\ell-1}\right)_{(r-1)(c-1), 1}+\left(A^{\ell}\right)_{(r-1)(c-1), 3} .
$$

By the induction hypothesis, the following inequalities hold:

$$
\left(A^{\ell-1}\right)_{r(c-1), c} \geq\left(A^{\ell-1}\right)_{(r-1)(c-1), 1},\left(A^{\ell}\right)_{r(c-1), c+2} \geq\left(A^{\ell}\right)_{(r-1)(c-1), 3} .
$$

Thus, we have the strict inequality $\left(A^{\ell+1}\right)_{r(c-1), c}>\left(A^{\ell+1}\right)_{(r-1)(c-1), 1}$. This causes the chain of strict inequalities

Finally, we have

$$
\begin{aligned}
& \left(A^{\ell+2}\right)_{r(c-1), 2(c-1)+1}>\left(A^{\ell+2}\right)_{(r-1)(c-1), c} \\
& \left(A^{\ell+3}\right)_{r(c-1), 3(c-1)+1}>\left(A^{\ell+3}\right)_{(r-1)(c-1), 2(c-1)+1} .
\end{aligned}
$$

$$
\left(A^{\ell+k-r}\right)_{r(c-1),(k-r+1)(c-1)+1}>\left(A^{\ell+k-r}\right)_{(r-1)(c-1),(k-r)(c-1)+1} .
$$

A similar argument can be used for the cases $j \equiv\{2,3, \ldots, c-2\}(\bmod (c-1))$.

Case 3: $t \not \equiv 0(\bmod (c-1))$ and $j \equiv 0(\bmod (c-1))$. Let $t \equiv 1(\bmod (c-1))$. Then

$$
\begin{aligned}
\left(A^{\ell+1}\right)_{t+c-1, j+c-1} & =\left(A^{\ell}\right)_{t+c-1, j+c-2}+\left(A^{\ell}\right)_{t+c-1, j+c-3}+\left(A^{\ell}\right)_{t+c-1, j+c}+\left(A^{\ell}\right)_{t+c-1, j+c+1}, \\
\left(A^{\ell+1}\right)_{t, j} & =\left(A^{\ell}\right)_{t, j-1}+\left(A^{\ell}\right)_{t, j-2}+\left(A^{\ell}\right)_{t, j+1}+\left(A^{\ell}\right)_{t, j+2} .
\end{aligned}
$$

By the induction hypothesis, we have:

$$
\begin{aligned}
\left(A^{\ell}\right)_{t+c-1, j+c-2} & \geq\left(A^{\ell}\right)_{t, j-1}, \\
\left(A^{\ell}\right)_{t+c-1, j+c} & \geq\left(A^{\ell}\right)_{t, j+1}, \quad \text { for } t+j+1 \leqslant k(c-1), \\
\left(A^{\ell}\right)_{t+c-1, j+c-3} & \geq\left(A^{\ell}\right)_{t, j-2}, \\
\left(A^{\ell}\right)_{t+c-1, j+c+1} & \geq\left(A^{\ell}\right)_{t, j+2}, \quad \text { for } t+j+2 \leqslant k(c-1) .
\end{aligned}
$$

Hence, we have $\left(A^{\ell+1}\right)_{t+c-1, j+c-1} \geq\left(A^{\ell+1}\right)_{t, j}$.

For the strict inequality, let $1 \leq r \leq k$ be a fixed number, for two rows $r(c-1)+1$ and $(r-1)(c-1)+1$ we have $\left(A^{\ell+1}\right)_{r(c-1)+1, c-1}=\left(A^{\ell}\right)_{r(c-1)+1, c-2}+\left(A^{\ell}\right)_{r(c-1)+1, c-3}+\left(A^{\ell}\right)_{r(c-1)+1, c}+\left(A^{\ell}\right)_{r(c-1)+1, c+1}$ and

$$
\left(A^{\ell+1}\right)_{(r-1)(c-1)+1,0}=\left(A^{\ell}\right)_{(r-1)(c-1)+1,1}+\left(A^{\ell}\right)_{(r-1)(c-1)+1,2} .
$$


Note that since $\Gamma(k)$ is symmetric we have

$$
\begin{aligned}
\left(A^{\ell}\right)_{r(c-1)+1, c-2} & =\left(A^{\ell}\right)_{r(c-1)+1, c-3}>0, \\
\left(A^{\ell}\right)_{r(c-1)+1, c} & =\left(A^{\ell}\right)_{r(c-1)+1, c+1}>0, \\
\left(A^{\ell}\right)_{r(c-1)+1,1} & =\left(A^{\ell}\right)_{r(c-1)+1,2}>0,
\end{aligned}
$$

for $\ell \geq r(c-1)$.

So,

$$
\left(A^{\ell+1}\right)_{r(c-1)+1, c-1}=2\left(A^{\ell}\right)_{r(c-1)+1, c-2}+2\left(A^{\ell}\right)_{r(c-1)+1, c+1}
$$

and

$$
\left(A^{\ell+1}\right)_{(r-1)(c-1)+1,0}=2\left(A^{\ell}\right)_{(r-1)(c-1)+1,2} .
$$

By the induction hypothesis, the following inequality holds:

$$
\left(A^{\ell}\right)_{r(c-1)+1, c+1} \geq\left(A^{\ell}\right)_{(r-1)(c-1)+1,2} .
$$

Thus, we have the strict inequality $\left(A^{\ell+1}\right)_{r(c-1)+1, c-1}>\left(A^{\ell+1}\right)_{(r-1)(c-1)+1,0}$. This causes the chain of strict inequalities

$$
\begin{aligned}
& \left(A^{\ell+2}\right)_{r(c-1)+1,2(c-1)}>\left(A^{\ell+2}\right)_{(r-1)(c-1)+1, c-1}, \\
& \left(A^{\ell+3}\right)_{r(c-1)+1,3(c-1)}>\left(A^{\ell+3}\right)_{(r-1)(c-1)+1,2(c-1)} .
\end{aligned}
$$

Finally, we have:

$$
\left(A^{\ell+(k-r+1)}\right)_{r(c-1)+1,(k-r+1)(c-1)-1}>\left(A^{\ell+(k-r+1)}\right)_{(r-1)(c-1)+1,(k-r)(c-1)-1} .
$$

A similar argument can be used for the cases $t \equiv\{2,3, \ldots, c-2\}(\bmod (c-1))$.

Case 4: $t \not \equiv 0(\bmod (c-1))$ and $j \equiv 1(\bmod (c-1))$. Let $t \equiv 1(\bmod (c-1))$, we have

$$
\begin{aligned}
\left(A^{\ell+1}\right)_{t+c-1, j+c-1} & =\left(A^{\ell}\right)_{t+c-1, j+c-2}+\left(A^{\ell}\right)_{t+c-1, j+c+1}, \\
\left(A^{\ell+1}\right)_{t, j} & =\left(A^{\ell}\right)_{t, j-1}+\left(A^{\ell}\right)_{t, j+2} .
\end{aligned}
$$

By the induction hypothesis, the following inequality holds:

$$
\left(A^{\ell}\right)_{t+c-1, j+c-2} \geq\left(A^{\ell}\right)_{t, j-1}, \quad\left(A^{\ell}\right)_{t+c-1, j+c+1} \geq\left(A^{\ell}\right)_{t, j+2} .
$$

Hence, we have $\left(A^{\ell}\right)_{t+c-1, j+c-1} \geq\left(A^{\ell}\right)_{t, j}$. For the strict inequality, let $1 \leq r \leq k$ be a fixed number, we consider two rows $r(c-1)+1$ and $(r-1)(c-1)+1$. Then

$$
\begin{aligned}
\left(A^{\ell+1}\right)_{r(c-1)+1, c}= & \left(A^{\ell}\right)_{r(c-1)+1, c-1}+\left(A^{\ell}\right)_{r(c-1)+1, c+2} \\
= & \left(A^{\ell-1}\right)_{r(c-1)+1, c-2}+\left(A^{\ell-1}\right)_{r(c-1)+1, c-3}+\left(A^{\ell-1}\right)_{r(c-1)+1, c} \\
& +\left(A^{\ell-1}\right)_{r(c-1)+1, c+1}+\left(A^{\ell}\right)_{r(c-1)+1, c+2}
\end{aligned}
$$

and

$$
\begin{aligned}
\left(A^{\ell+1}\right)_{(r-1)(c-1)+1,1} & =\left(A^{\ell}\right)_{(r-1)(c-1)+1,0}+\left(A^{\ell}\right)_{(r-1)(c-1)+1,3} \\
& =\left(A^{\ell-1}\right)_{(r-1)(c-1)+1,1}+\left(A^{\ell-1}\right)_{(r-1)(c-1)+1,2}+\left(A^{\ell}\right)_{(r-1)(c-1)+1,3} .
\end{aligned}
$$


Note that since $\Gamma(k)$ is symmetric, $\left(A^{\ell-1}\right)_{r(c-1)+1, c-2}=\left(A^{\ell-1}\right)_{r(c-1)+1, c-3}>0$, $\left(A^{\ell-1}\right)_{r(c-1)+1, c}=\left(A^{\ell-1}\right)_{r(c-1)+1, c+1}>0$ and $\left(A^{\ell-1}\right)_{r(c-1)+1,1}=\left(A^{\ell-1}\right)_{r(c-1)+1,2}>0$, for $\ell \geq r(c-1)$. So,

$$
\left(A^{\ell+1}\right)_{r(c-1)+1, c}=2\left(A^{\ell-1}\right)_{r(c-1)+1, c-2}+2\left(A^{\ell-1}\right)_{r(c-1)+1, c}+\left(A^{\ell}\right)_{r(c-1)+1, c+2}
$$

and

$$
\left(A^{\ell+1}\right)_{(r-1)(c-1)+1,1}=2\left(A^{\ell-1}\right)_{(r-1)(c-1)+1,1}+\left(A^{\ell}\right)_{(r-1)(c-1)+1,3} .
$$

By the induction hypothesis, the following inequalities hold:

$$
\left(A^{\ell-1}\right)_{r(c-1)+1, c} \geqslant\left(A^{\ell-1}\right)_{(r-1)(c-1)+1,1},\left(A^{\ell}\right)_{r(c-1)+1, c+2} \geqslant\left(A^{\ell}\right)_{(r-1)(c-1)+1,3} .
$$

Thus, we have the strict inequality $\left(A^{\ell+1}\right)_{r(c-1)+1, c}>\left(A^{\ell+1}\right)_{(r-1)(c-1)+1,1}$. This causes the chain of strict inequalities

$$
\begin{aligned}
& \left(A^{\ell+2}\right)_{r(c-1)+1,2(c-1)+1}>\left(A^{\ell+2}\right)_{(r-1)(c-1)+1, c}, \\
& \left(A^{\ell+3}\right)_{r(c-1)+1,3(c-1)+1}>\left(A^{\ell+3}\right)_{(r-1)(c-1)+1,2(c-1)+1} .
\end{aligned}
$$

Finally,

$$
\left(A^{\ell+k-r}\right)_{r(c-1)+1,(k-r+1)(c-1)+1}>\left(A^{\ell+k-r}\right)_{(r-1)(c-1)+1,(k-r)(c-1)+1} .
$$

A similar argument can be used for $t \equiv r \in\{2,3, \ldots, c-2\}(\bmod (c-1))$.

The number of closed walks of length $\ell$ starting at the vertex $v_{t}$ is equal to the entry $(t, t)$ in matrix $A^{\ell}$. Therefore,

$$
S_{\ell}(k(c-1), t+(c-1))=\left(A^{\ell}\right)_{t+(c-1), t+(c-1)} .
$$

By the induction hypothesis, we conclude that $S_{\ell}(k(c-1), t+(r-1)(c-1)) \leq$ $S_{\ell}(k(c-1), t+r(c-1))$ for all $0 \leq t \leq c-1$ and $r \leq\left[\frac{k}{2}\right](c-1)$. Hence the strict inequality holds when $\ell \geq\left[\frac{k}{2}\right]$.

\section{The Minimum Estrada Index of $\Gamma(k)$}

Let $G^{\prime}$ be a point attaching strict $k_{1}$-quasi tree graph of even length $c$ and $\delta \in V\left(G^{\prime}\right)$. For $k-k_{1}=k_{2}$, let $G^{\prime}\left(\left\lfloor\frac{k_{2}}{2}\right\rfloor,\left\lceil\frac{k_{2}}{2}\right\rceil\right)$ be the graph obtained from $G^{\prime}$ by attaching two graphs $\Gamma\left(\left\lfloor\frac{k_{2}}{2}\right\rfloor\right)$ and $\Gamma\left(\left\lceil\frac{k_{2}}{2}\right\rceil\right)$ at $\delta$.

Let $N_{\ell}\left(G^{\prime}\left(\left\lfloor\frac{k_{2}}{2}\right\rfloor(c-1),\left\lceil\frac{k_{2}}{2}\right\rceil(c-1) ; \delta\right)\right.$ (respectively, $N_{\ell}\left(G^{\prime}\left(\left\lfloor\frac{k_{2}}{2}\right\rfloor(c-1)+c-1\right.\right.$, $\left.\left.\left\lceil\frac{k_{2}}{2}\right\rceil(c-1)-c+1\right) ; \delta\right)$ be the set of $(\delta, \delta)$-walks of length $\ell$ in $G^{\prime}\left(\left\lfloor\frac{k_{2}}{2}\right\rfloor(c-1),\left\lceil\frac{k_{2}}{2}\right\rceil(c-1)\right)$ (respectively, $G^{\prime}\left(\left\lfloor\frac{k_{2}}{2}\right\rfloor(c-1)+c-1,\left\lceil\frac{k_{2}}{2}\right\rceil(c-1)-c+1\right)$ starting and ending at the edges or only one edge in $G^{\prime}$ and let $N_{\ell}^{\prime}\left(G^{\prime}\left(\left\lfloor\frac{k_{2}}{2}\right\rfloor(c-1),\left\lceil\frac{k_{2}}{2}\right\rceil(c-1)\right) ; \delta\right)$ (respectively, $\left.N_{\ell}^{\prime}\left(G^{\prime}\left(\left\lfloor\frac{k_{2}}{2}\right\rfloor(c-1)+c-1,\left\lceil\frac{k_{2}}{2}\right\rceil(c-1)-c+1\right) ; \delta\right)\right)$ be the set of $(\delta, \delta)$-walks of length $\ell$ in $G^{\prime}\left(\left\lfloor\frac{k_{2}}{2}\right\rfloor(c-1),\left\lceil\frac{k_{2}}{2}\right\rceil(c-1)\right)$ (respectively, $G^{\prime}\left(\left\lfloor\frac{k_{2}}{2}\right\rfloor(c-1)+c-1,\left\lceil\frac{k_{2}}{2}\right\rceil(c-1)-c+1\right)$ starting and ending at the edges or only one edge in union $\Gamma\left(\left\lfloor\frac{k_{2}}{2}\right\rfloor\right) \cup \Gamma\left(\left\lceil\frac{k_{2}}{2}\right\rceil\right.$ ) (respectively, $\left.\Gamma\left(\left\lfloor\frac{k_{2}}{2}\right\rfloor+1\right) \cup \Gamma\left(\left\lceil\frac{k_{2}}{2}\right\rceil-1\right)\right)$. 
In the following let $G^{\prime}\left(\left\lfloor\frac{k_{2}}{2}\right\rfloor(c-1),\left\lceil\frac{k_{2}}{2}\right\rceil(c-1)\right):=G(1)$ and let $G^{\prime}\left(\left\lfloor\frac{k_{2}}{2}\right\rfloor(c-1)+c-1\right.$, $\left.\left\lceil\frac{k_{2}}{2}\right\rceil(c-1)-c+1\right):=G(2)$. By our definition, both graphs $\Gamma\left(\left\lfloor\frac{k_{2}}{2}\right\rfloor\right) \cup \Gamma\left(\left\lceil\frac{k_{2}}{2}\right\rceil\right)$ and $\Gamma\left(\left\lfloor\frac{k_{2}}{2}\right\rfloor+1\right) \cup \Gamma\left(\left\lceil\frac{k_{2}}{2}\right\rceil-1\right)$ are isomorphic to $\Gamma\left(k_{2}\right)$, so they are denoted by $\Gamma\left(k_{2}\right)$.

Lemma 4.1. If $\left\lfloor\frac{k_{2}}{2}\right\rfloor \geq 1$, then for positive integer $\ell$,

(i) $\left.\left|N_{\ell}\left(G^{\prime}(2) ; \delta\right)\right| \leq \mid N_{\ell}\left(G^{\prime}(1)\right) ; \delta\right) \mid$;

(ii) $\left.\left|N_{\ell}^{\prime}\left(G^{\prime}(2) ; \delta\right)\right| \leq \mid N_{\ell}^{\prime}\left(G^{\prime}(1)\right) ; \delta\right) \mid$.

Proof. Let $\omega \in N_{\ell}\left(G^{\prime}(2) ; \delta\right)$, we may decompose $\omega$ into maximal sections in union $\Gamma\left(\left\lfloor\frac{k_{2}}{2}\right\rfloor+1\right) \cup \Gamma\left(\left\lceil\frac{k_{2}}{2}\right\rceil-1\right)$ or in $G^{\prime}$. Each of them is one of the following types.

(Type 1): a $(\delta, \delta)$ - walk in union $\Gamma\left(\left\lfloor\frac{k_{2}}{2}\right\rfloor+1\right) \cup \Gamma\left(\left\lceil\frac{k_{2}}{2}\right\rceil-1\right)$.

(Type 2): a walk in $G^{\prime}(2)$ with all edges in $G^{\prime}$.

Similarly, we may decompose any $\omega \in N_{\ell}\left(G^{\prime}(1) ; \delta\right)$ into maximal sections in $G^{\prime}$ or in union $\Gamma\left(\left\lfloor\frac{k_{2}}{2}\right\rfloor\right) \cup \Gamma\left(\left\lceil\frac{k_{2}}{2}\right\rceil\right)$. Each of them is one of the following types.

(Type 3): a $(\delta, \delta)$ - walk in union $\Gamma\left(\left\lfloor\frac{k_{2}}{2}\right\rfloor\right) \cup \Gamma\left(\left\lceil\frac{k_{2}}{2}\right\rceil\right)$.

(Type 4): a walk in $G^{\prime}(1)$ with all edges in $G^{\prime}$.

Next, for any $\omega \in N_{\ell}\left(G^{\prime}(2) ; \delta\right)$, we can replace the even indices by the odd indices that are in front of each other see Figure 2. Hence, from now on, $\omega$ is a $(\delta, \delta)$ - walk with

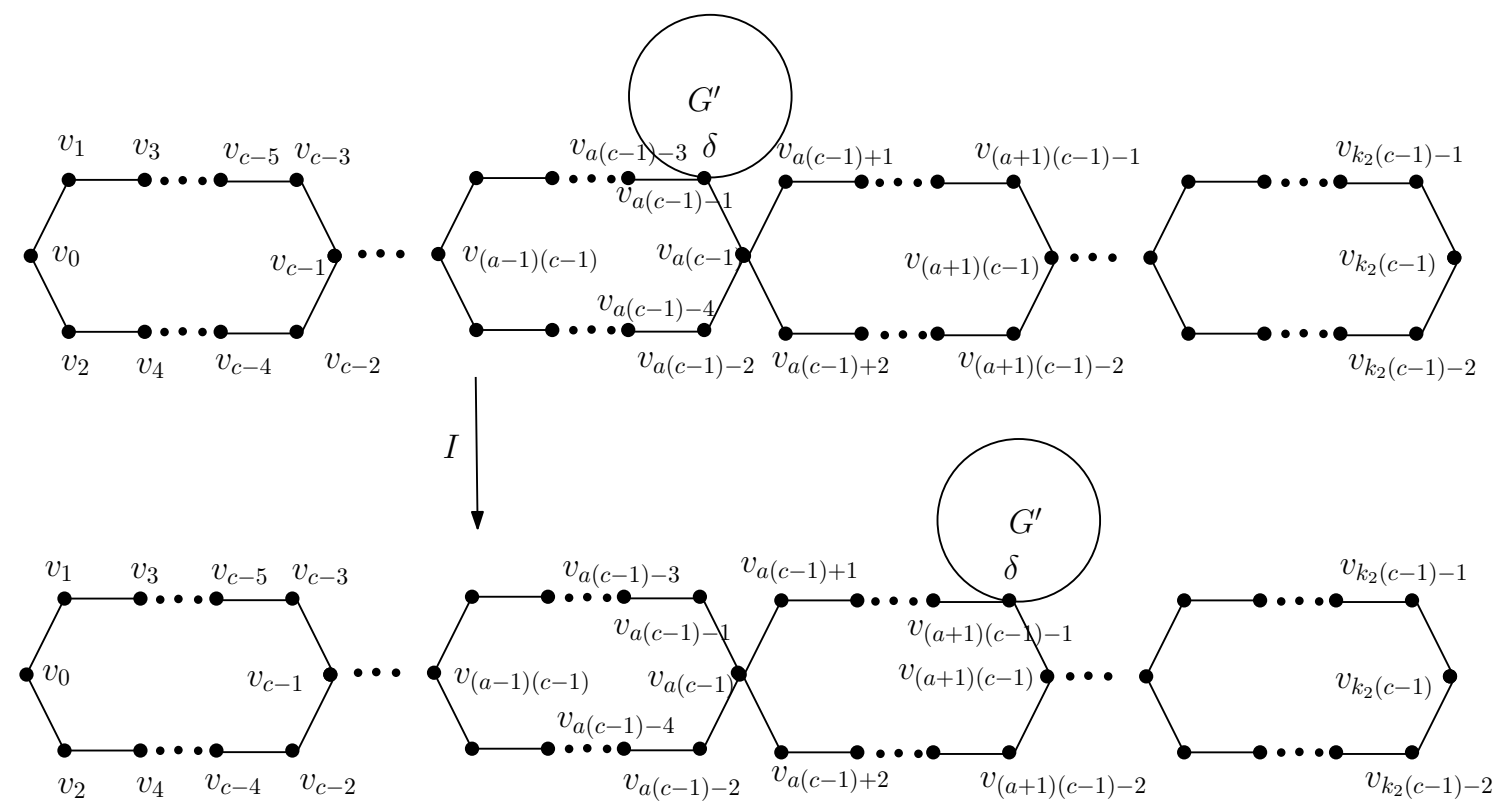

FiguRE 2. Transformation $I$.

only odd or even indices. So $\omega$ is a $(\delta, \delta)$ - walk with odd indices. By Lemma 3.2 there is an injection mapping $\xi_{s^{\prime}}^{1}$ that is a $(\delta, \delta)$ - walk of length $s^{\prime}$ in $\Gamma\left(\left\lfloor\frac{k_{2}}{2}\right\rfloor+1\right) \cup \Gamma\left(\left\lceil\frac{k_{2}}{2}\right\rceil-1\right)$ into a $(\delta, \delta)$ - walk of length $s^{\prime}$ in $\Gamma\left(\left\lfloor\frac{k_{2}}{2}\right\rfloor\right) \cup \Gamma\left(\left\lceil\frac{k_{2}}{2}\right\rceil\right)$.

Let $\omega^{\prime}=\omega_{1} \omega_{2} \omega_{3} \cdots \in N_{\ell}\left(\Gamma\left(k_{2}\right)\right)$, where $\omega_{i}$ is a walk of length $s_{i}^{\prime}$ of type (1) or (2) for $i \geq 1$. Let $\xi^{\star}\left(\omega^{\prime}\right)=\xi^{\star}\left(\omega_{1}\right) \xi^{\star}\left(\omega_{2}\right) \cdots$, where $\xi^{\star}\left(\omega_{i}\right)=\xi_{s_{i}^{\prime}}^{1}\left(\omega_{i}\right)$ and $\xi^{\star}\left(\omega_{i}\right)=\omega_{i}$ if $\omega_{i}$ 
is of type 2 so $\xi^{\star}\left(\omega_{i}\right)$ for $i \geq 1$ is of type 3 or 4 and thus $\xi^{\star}\left(\omega^{\prime}\right) \in N_{\ell}\left(G^{\prime}(1)\right)$. Thus $\left|N_{\ell}\left(G^{\prime}(2) ; \delta\right)\right| \leqslant\left|N_{\ell}\left(G^{\prime}(1) ; \delta\right)\right|$. This prove (i). The proof for (ii) is similar.

Theorem 4.1. If $\left\lfloor\frac{k_{2}}{2}\right\rfloor \geq 1$, then $S_{\ell}\left(G^{\prime}(2)\right) \leq S_{\ell}\left(G^{\prime}(1)\right)$. For $\ell \geq\left[\frac{k_{2}}{2}\right](c-1)$, the strict inequality holds.

Proof. Let $B_{1}$ and $B_{2}$ be the sets of closed walks of length $\ell$ in $G^{\prime}(1)$ and $G^{\prime}(2)$ respectively, containing some edges in $G^{\prime}$. Then $S_{\ell}\left(G^{\prime}(2)\right)=S_{\ell}\left(\Gamma\left(\left\lfloor\frac{k_{2}}{2}\right\rfloor+1\right) \cup \Gamma\left(\left\lceil\frac{k_{2}}{2}\right\rceil-\right.\right.$ $1))+\left|B_{2}\right|$ and $S_{\ell}\left(G^{\prime}(1)\right)=S_{\ell}\left(\Gamma\left(\left\lfloor\frac{k_{2}}{2}\right)\right\rfloor \cup \Gamma\left(\left\lceil\frac{k_{2}}{2}\right\rceil\right)\right)+\left|B_{1}\right|$. Since $\Gamma\left(\left\lfloor\frac{k_{2}}{2}\right\rfloor+1\right) \cup \Gamma\left(\left\lceil\frac{k_{2}}{2}\right\rceil-1\right)$ and $\Gamma\left(\left\lfloor\frac{k_{2}}{2}\right\rfloor\right) \cup \Gamma\left(\left\lceil\frac{k_{2}}{2}\right\rceil\right)$ are isomorphic to $\Gamma\left(k_{2}\right)$, we only need to prove that $\left|B_{2}\right| \leqslant\left|B_{1}\right|$ for all $\ell \geqslant 0$. Let $B_{21}$ and $B_{22}$ be two subsets of $B_{2}$ for which every closed walk starts at a vertex in $V\left(\Gamma\left(\left\lfloor\frac{k_{2}}{2}\right\rfloor+1\right) \cup \Gamma\left(\left\lceil\frac{k_{2}}{2}\right\rceil-1\right)\right)$ and $V\left(G^{\prime}\right)-\{\delta\}$, respectively. Then $\left|B_{2}\right|=\left|B_{21}\right|+\left|B_{22}\right|$. Let $B_{11}$ and $B_{12}$ be two subsets of $B_{1}$ for which every closed walk starts at a vertex in $V\left(\Gamma\left(\left\lfloor\frac{k_{2}}{2}\right\rfloor\right) \cup \Gamma\left(\left\lceil\frac{k_{2}}{2}\right\rceil\right)\right)$ and $V\left(G^{\prime}\right)-\{\delta\}$, respectively. Then $\left|B_{1}\right|=\left|B_{11}\right|+\left|B_{12}\right|$.

We may decompose any $\omega \in B_{21}$ into three parts $\omega_{1} \omega_{2} \omega_{3}$, where $\omega_{1}, \omega_{3}$ are walks in $\Gamma\left(\left\lfloor\frac{k_{2}}{2}\right\rfloor+1\right) \cup \Gamma\left(\left\lceil\frac{k_{2}}{2}\right\rceil-1\right)$ and $\omega_{2}$ is the longest walk of $\omega$ in $G^{\prime}(2)$ starting and ending at the edges or only one edge in $G^{\prime}$. By the choice of $\omega_{2}$, we have that $\omega_{2}$ is a $(\delta, \delta)$ walk. Let $B_{21}(\omega, \ell)=\left\{\omega \in B_{21}: \omega_{2}\right.$ is a $(\delta, \delta)$ - walk $\}$. Thus $\left|B_{21}\right|=\left|B_{21}(\omega, \ell)\right|$. Let $B_{11}(\omega, \ell)=\left\{\omega \in B_{11}: \omega_{2}\right.$ is a $(\delta, \delta)$-walk $\}$. So $\left|B_{11}\right|=\left|B_{11}(\omega, \ell)\right|$.

Let $V\left(\Gamma\left(\left\lfloor\frac{k_{2}}{2}\right\rfloor+1\right) \cup \Gamma\left(\left\lceil\frac{k_{2}}{2}\right\rceil-1\right)\right):=V(2)$. Then

$$
\begin{aligned}
\left|B_{21}(\omega, \ell)\right|= & \sum_{\substack{\ell_{1}+\ell_{2}+\ell_{3}=\ell \\
\ell_{1}, \ell_{3} \geq 0, \ell_{2} \geq 2}} \sum_{\beta \in V(2)} S_{\ell_{1}}\left(\Gamma\left(\left\lfloor\frac{k_{2}}{2}\right\rfloor+1\right) \cup \Gamma\left(\left\lceil\frac{k_{2}}{2}\right\rceil-1\right) ; \beta, \delta\right) \\
& \times\left|N_{\ell_{2}}\left(G^{\prime}(2) ; \delta\right)\right| S_{\ell_{3}}\left(\Gamma\left(\left\lfloor\frac{k_{2}}{2}\right\rfloor+1\right) \cup \Gamma\left(\left\lceil\frac{k_{2}}{2}\right\rceil-1\right) ; \delta, \beta\right) \\
= & \sum_{\substack{\ell_{1}+\ell_{2}+\ell_{3}=\ell \\
\ell_{1}, \ell_{3} \geqslant 0, \ell_{2} \geqslant 2}}\left|N_{\ell_{2}}\left(G^{\prime}(2) ; \delta\right)\right| \\
& \times \sum_{\beta \in V(2)} S_{\ell_{1}}\left(\Gamma\left(\left\lfloor\frac{k_{2}}{2}\right\rfloor+1\right) \cup \Gamma\left(\left\lceil\frac{k_{2}}{2}\right]-1\right) ; \beta, \delta\right) \\
& \left.\times S_{\ell_{3}}\left(\Gamma\left(\mid \frac{k_{2}}{2}\right\rfloor+1\right) \cup \Gamma\left(\left\lceil\frac{k_{2}}{2}\right\rfloor-1\right) ; \delta, \beta\right) \\
= & \left.\sum_{\substack{\ell_{1}+\ell_{2}+\ell_{3}=\ell \\
\ell_{1}, \ell_{3} \geq 0, \ell_{2} \geq 2}}\left|N_{\ell_{2}}\left(G^{\prime}(2) ; \delta\right)\right| . S_{\ell_{1}+\ell_{3}}\left(\Gamma\left(\mid \frac{k_{2}}{2}\right\rfloor+1\right) \cup \Gamma\left(\left\lceil\frac{k_{2}}{2}\right\rceil-1\right) ; \delta\right) .
\end{aligned}
$$

Similarly,

$$
\left|B_{21}(\omega, \ell)\right|=\sum_{\substack{\ell_{1}+\ell_{2}+\ell_{3}=\ell \\ \ell_{1}, \ell_{3} \geqslant 0, \ell_{2} \geqslant 2}}\left|N_{\ell_{2}}\left(G^{\prime}(1) ; \delta\right)\right| S_{\ell_{1}+\ell_{3}}\left(\Gamma\left(\left\lfloor\frac{k_{2}}{2}\right\rfloor+1\right) \cup \Gamma\left(\left\lceil\frac{k_{2}}{2}\right\rceil-1\right) ; \delta\right) .
$$


By Lemma 4.1, we have $\left|N_{\ell_{2}}\left(G^{\prime}(2) ; \delta\right)\right| \leq\left|N_{\ell_{2}}\left(G^{\prime}(1) ; \delta\right)\right|$ for all positive integers $\ell_{2}$ and by Lemma 3.2, we have $\left.S_{t}\left(\Gamma\left(\left\lfloor\frac{k_{2}}{2}\right\rfloor+1\right) \cup \Gamma\left(\left\lceil\frac{k_{2}}{2}\right\rceil-1\right)\right) ; \delta\right) \leq S_{t}\left(\Gamma\left(\left\lfloor\frac{k_{2}}{2}\right\rfloor\right) \cup \Gamma\left(\left\lceil\frac{k_{2}}{2}\right\rceil\right) ; \delta\right)$ for all positive integers $t$. Thus $\left|B_{21}(\omega, \ell)\right| \leq\left|B_{11}(\omega, \ell)\right|$. Note that this inequality is strict for some positive integer $\ell_{0}=t_{0}+c-1$ where $t_{0} \geq \frac{k_{2}}{2}$. Also $\left|B_{21}\right| \leq\left|B_{11}\right|$ for all positive integers $\ell$, and it is strict for some positive integer $\ell_{0}$.

By a similar argument as above, we can prove that $\left|B_{22}\right| \leq\left|B_{12}\right|$. Thus $\left|B_{2}\right| \leq\left|B_{1}\right|$ for all positive integers $\ell$, and it is strict for some positive integer $\ell_{0}$.

Lemma 4.2. For all integer $\ell>c, k \geq 2$, we have

$$
S_{\ell}(k(c-1), 2) \leq S_{\ell}(k(c-1), 4) \leq \cdots \leq S_{\ell}(k(c-1), c / 2-2), S_{\ell}(k(c-1), c / 2) .
$$

Proof. First, we show that every diagonal parallel to the main diagonal and the main diagonal are unimodal. Let $H$ be the subgraph of $\Gamma(k)$ with vertex set $\left\{v_{0}, v_{1}, \ldots, v_{c}-1\right\}$. By Lemma 3.1, we only need to show that the diagonals parallel to the main diagonal increase for $s+j \leq c-1$. Let $s$ be an even integer. For the odd integer the proof is similar. Using induction on integer $\ell$, we will prove that $\left(A^{\ell}\right)_{s+2, j+2} \geq\left(A^{\ell}\right)_{s, j}$ for all $0 \leq s, j \leq c-2$ with $s+j \leq c-1$.

Note that by the definition of $\Gamma(k)$, two vertices $v_{s}$ and $v_{j}$ are adjacent if and only if $v_{s+2}$ and $v_{j+2}$ are adjacent.

We have the following cases.

Case 1: $j \equiv 0(\bmod 2)$ and $j \neq 0$. Then

$$
\begin{aligned}
\left(A^{\ell+1}\right)_{s+2, j+2} & =\left(A^{\ell}\right)_{s+2, j}+\left(A^{\ell}\right)_{s+2, j+4}, \\
\left(A^{\ell+1}\right)_{s, j} & =\left(A^{\ell}\right)_{s, j-2}+\left(A^{\ell}\right)_{s, j+2} .
\end{aligned}
$$

By the induction hypothesis, we have the following results:

$$
\begin{aligned}
\left(A^{\ell}\right)_{s+2, j} & \geq\left(A^{\ell}\right)_{s, j-2}, \\
\left(A^{\ell}\right)_{s+2, j+4} & \geq\left(A^{\ell}\right)_{s, j+2}, \quad \text { for } s+j+4 \leq c-1 .
\end{aligned}
$$

Hence, we have $\left(A^{\ell}\right)_{s+2, j+2} \geq\left(A^{\ell}\right)_{s, j}$. Since, there is a closed walk of length $c$ starting from $v_{0}$ which is not including the edge $v_{c} v_{c+1}$, the inequality is strict for $\ell>c$.

Case $2: j \equiv 1(\bmod 2)$. The proof is similar to Case 1 .

The number of closed walks of length $\ell$ starting at the even vertex $v_{s}$ is equal to the entry $(s, s)$ in matrix $A^{\ell}$,

$$
S_{\ell}(c-1, s)=\left(A^{\ell}\right)_{s, s} .
$$

By induction hypothesis, we can conclude that $S_{\ell}(c-1, s) \leq S_{\ell}(c-1, s+2)$ for every $0<s<c-1$. Note that the strict inequality holds when $\ell \geq \frac{c}{2}$.

Let $G$ be a point attaching strict $k_{1}$-quasi tree graph of even length $c$ and $\alpha \in V(G)$ and let $C_{c}$ be the cycle $H$ of $\Gamma(k)$ with $k_{2}$ cycles where $k_{1}+k_{2}=k$. We decompose $C_{c}$ into two paths denote by $P_{\frac{c}{2}}$ and $Q_{\frac{c}{2}}$, having common vertices in initial and final. Let $G\left(\frac{c}{2}, \frac{c}{2}\right)$ be the graph obtained from $G$ by attaching $P_{\frac{c}{2}}$ and $Q_{\frac{c}{2}}$ at $\alpha$ in $G$. 


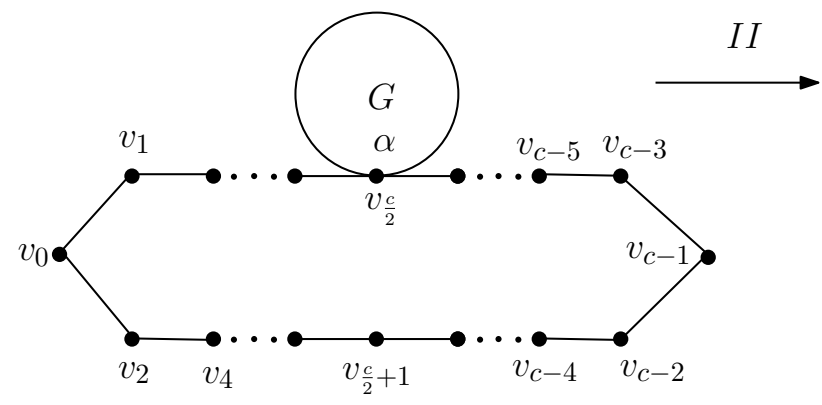

$G(1)$

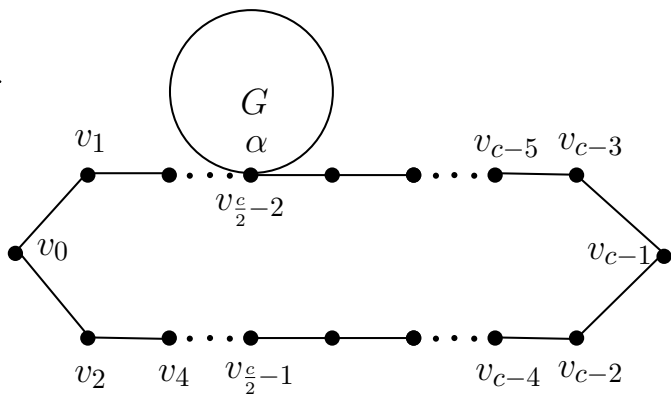

$G(2)$

FiguRE 3. Transformation $I I$.

Let $M_{\ell}\left(G\left(\frac{c}{2}, \frac{c}{2}\right) ; \alpha\right)$ (respectively $M_{\ell}\left(G\left(\frac{c}{2}+2, \frac{c}{2}-2\right) ; \alpha\right)$ ) be the set of $(\alpha, \alpha)$-walks of length $\ell$ in $G\left(\frac{c}{2}, \frac{c}{2}\right)$ (respectively $G\left(\frac{c}{2}+2, \frac{c}{2}-2\right)$ ) starting and ending at the edges or only one edge in $G$ and let $M_{\ell}^{\prime}\left(G\left(\frac{c}{2}, \frac{c}{2}\right) ; \alpha\right)$ (respectively $M_{\ell}^{\prime}\left(G\left(\frac{c}{2}+2, \frac{c}{2}-2\right) ; \alpha\right)$ ) be the set of $(\alpha, \alpha)$-walks of length $\ell$ in $G\left(\frac{c}{2}, \frac{c}{2}\right)$ (respectively $G\left(\frac{c}{2}+2, \frac{c}{2}-2\right)$ ), starting and ending at the edges or only one edge in $P_{\frac{c}{2}} \cup Q_{\frac{c}{2}}$ (respectively $P_{\frac{c}{2}+1} \cup Q_{\frac{c}{2}-1}$ ). In the following let $G\left(\frac{c}{2}, \frac{c}{2}\right):=G(1)$ and $G\left(\frac{c}{2}+2, \frac{c}{2}-2\right):=G(2)$. By definition $P_{\frac{c}{2}} \cup Q_{\frac{c}{2}}$ and $P_{\frac{c}{2}+1} \cup Q_{\frac{c}{2}-1}$ are isomorphic to $C_{1}$, so we denoted them by $C_{1}$.

Lemma 4.3. Let $c$ be an even integer. If $\ell \geq \frac{c}{2}$, then

(i) $\left.\left|M_{\ell}(G(2) ; \alpha)\right| \leq \mid M_{\ell}(G(1)) ; \alpha\right) \mid$;

(ii) $\left.\left|M_{\ell}^{\prime}(G(2) ; \alpha)\right| \leq \mid M_{\ell}^{\prime}(G(1)) ; \alpha\right) \mid$.

Proof. Let $\omega \in M_{\ell}(G(2) ; \alpha)$, we may decompose $\omega$ into maximal sections in union $P_{\frac{c}{2}+1} \cup Q_{\frac{c}{2}-1}$ or in $G$. Each of them is one of the following types.

(1) a $(\alpha, \alpha)$ - walk in union $P_{\frac{c}{2}+1} \cup Q_{\frac{c}{2}-1}$.

(2) a walk in $G(2)$ with all edges in $G$.

Similarly, we may decompose any $\omega \in M_{\ell}(G(1) ; \alpha)$ into maximal sections in union $P_{\frac{c}{2}} \cup Q_{\frac{c}{2}}$ or in $G$. Each of these maximal sections has one of the following types.

(3) a $(\alpha, \alpha)$-walk in union $P_{\frac{c}{2}} \cup Q_{\frac{c}{2}}$.

(4) a walk in $G(1)$ with all edges in $G$.

Next, since $\Gamma(k)$ is symmetric, for any $\omega \in M_{\ell}(G(2) ; \alpha)$, we can replace the even indices with the odd indices that are in front of each other see Figure 3. Hence, from now on, $\omega$ is a $(\alpha, \alpha)$ - walk with only odd or even indices. So without loss of generality $\omega$ is a $(\alpha, \alpha)$-walk with only odd indices. By definition, two unions $P_{\frac{c}{2}} \cup Q_{\frac{c}{2}}$ and $P_{\frac{c}{2}+1} \cup Q_{\frac{c}{2}-1}$ are isomorphic to $C_{1}$ and by Lemma 4.2 there exists an injection mapping $\eta_{\ell}^{1}$ from a $(\alpha, \alpha)$-walk of length $\ell$ in $P_{\frac{c}{2}+1} \cup Q_{\frac{c}{2}-1}$ into a $(\alpha, \alpha)$ - walk of length $\ell$ in $P_{\frac{c}{2}} \cup Q_{\frac{c}{2}}$. Let $\omega=\omega_{1} \omega_{2} \omega_{3} \cdots \in M_{\ell}\left(P_{\frac{c}{2}+1} \cup Q_{\frac{c}{2}-1}\right)$, where $\omega_{i}$ is a walk of length $\ell_{i}$ of type (1) or (2) for $i \geq 1$. Let $\eta^{\star}(\omega)=\eta^{\star}\left(\omega_{1}\right) \eta^{\star}\left(\omega_{2}\right) \ldots$ where $\eta^{\star}\left(\omega_{i}\right)=\eta_{\ell_{i}}^{1}\left(\omega_{i}\right)$ and $\eta^{\star}\left(\omega_{i}\right)=\omega_{i}$ if $\omega_{i}$ is type (2) so $\eta^{\star}\left(\omega_{i}\right)$ for $i \geq 1$ is of type (3) or (4) and thus 
$\eta^{\star}(\omega) \in M_{\ell}(G(1))$. Thus, $\left|M_{\ell}(G(2) ; \alpha)\right| \leq\left|M_{\ell}(G(1) ; \alpha)\right|$. This prove (i). The proof of (ii) is similar.

Theorem 4.2. Let $c$ be an even integer. If $\frac{c}{2} \geq 3$, then $S_{\ell}(G(2)) \leq S_{\ell}(G(1))$. For $\ell>\frac{c}{2}$, the strict inequality holds.

Proof. Let $A_{1}$ and $A_{2}$ be two sets of closed walks of length $\ell$ in $G(1)$ and $G(2)$, respectively, containing some edges in $G$. Then $S_{\ell}(G(2))=S_{\ell}\left(P_{\frac{c}{2}+1} \cup Q_{\frac{c}{2}-1}\right)+\left|A_{2}\right|$ and $S_{\ell}(G(1))=S_{\ell}\left(P_{\frac{c}{2}} \cup Q_{\frac{c}{2}}\right)+\left|A_{1}\right|$.

By our definition, $P_{\frac{c}{2}} \cup Q_{\frac{c}{2}}$ and $P_{\frac{c}{2}+1} \cup Q_{\frac{c}{2}-1}$ are isomorphic to $C_{1}$, and we need only to prove that $\left|A_{2}\right| \leq\left|A_{1}\right|$ for all $\ell \geq 0$.

Let $A_{21}$ and $A_{22}$ be two subsets of $A_{2}$ for which every closed walk starts at a vertex in $V\left(P_{\frac{c}{2}+1} \cup Q_{\frac{c}{2}-1}\right)$ and in $V(G)-\{\alpha\}$, respectively. Then $\left|A_{2}\right|=\left|A_{21}\right|+\left|A_{22}\right|$.

Let $A_{11}$ and $A_{12}$ be two subsets of $A_{1}$ for which every closed walk starts at a vertex in $V\left(P_{\frac{c}{2}} \cup Q_{\frac{c}{2}}\right)$ and in $V(G)-\{\alpha\}$, respectively. Then $\left|A_{1}\right|=\left|A_{11}\right|+\left|A_{12}\right|$.

We may decompose any $\omega \in A_{21}$ into three sections $\omega_{1} \omega_{2} \omega_{3}$, where $\omega_{1}, \omega_{3}$ are walks in $P_{\frac{c}{2}+1} \cup Q_{\frac{c}{2}-1}$ and $\omega_{2}$ is the longest walk of $\omega$ in $G(2)$ starting and ending at the edges in $G$. By the choice of $\omega_{2}$, we have that $\omega_{2}$ is a $(\alpha, \alpha)$-walk. Let $A_{21}(\omega, \ell)=\left\{\omega \in A_{21}: \omega_{2}\right.$ is a $(\alpha, \alpha)$-walk $\}$. So, we have $\left|A_{21}\right|=\left|A_{21}(\omega, \ell)\right|$.

Let $A_{11}(\omega, \ell)=\left\{\omega \in A_{11}: \omega_{2}\right.$ is a $(\alpha, \alpha)$-walk $\}$. So, we have $\left|A_{11}\right|=\left|A_{11}(\omega, \ell)\right|$.

Let $V\left(P_{\frac{c}{2}+1} \cup Q_{\frac{c}{2}-1}\right):=V(1)$. Let $t=\left|M_{\ell_{2}}(G(2) ; \alpha)\right|$. From this decomposition for $\omega \in A_{21}$ and by the definition of $A_{21}(\omega, \ell)$, we have

$$
\begin{aligned}
\left|A_{21}(\omega, \ell)\right|= & \sum_{\substack{\ell_{1}+\ell_{2}+\ell_{3}=\ell \\
\ell_{1}, \ell_{3} \geqslant 0, \ell_{2} \geqslant 2}} \sum_{\beta \in V(1)} S_{\ell_{1}}\left(P_{\frac{c}{2}+1} \cup Q_{\frac{c}{2}-1} ; \beta, \alpha\right) \cdot t \cdot S_{\ell_{3}}\left(P_{\frac{c}{2}+1} \cup Q_{\frac{c}{2}-1} ; \alpha, \beta\right) \\
= & \sum_{\substack{\ell_{1}+\ell_{2}+\ell_{3}=\ell \\
\ell_{1}, \ell_{3} \geqslant 0, \ell_{2} \geqslant 2}} t \cdot \sum_{\beta \in V(1)} S_{\ell_{1}}\left(P_{\frac{c}{2}+1} \cup Q_{\frac{c}{2}-1} ; \beta, \alpha\right) . S_{\ell_{3}}\left(P_{\frac{c}{2}+1} \cup Q_{\frac{c}{2}-1} ; \alpha, \beta\right) \\
= & \sum_{\substack{\ell_{1}+\ell_{2}+\ell_{3}=\ell \\
\ell_{1}, \ell_{3} \geqslant 0, \ell_{2} \geqslant 2}} \text { t. } S_{\ell_{1}+\ell_{3}}\left(P_{\frac{c}{2}+1} \cup Q_{\frac{c}{2}-1} ; \alpha\right) .
\end{aligned}
$$

Similarly,

$$
\left|A_{21}(\omega, \ell)\right|=\sum_{\substack{\ell_{1}+\ell_{2}+\ell_{3}=\ell \\ \ell_{1}, \ell_{3} \geqslant 0, \ell_{2} \geqslant 2}}\left|M_{\ell_{2}}(G(1) ; \alpha)\right| . S_{\ell_{1}+\ell_{3}}\left(P_{\frac{c}{2}} \cup Q_{\frac{c}{2}} ; \alpha\right) .
$$

By Lemma 4.3, we have $\left|M_{\ell_{2}}(G(2) ; \alpha)\right| \leq\left|M_{\ell_{2}}(G(1) ; \alpha)\right|$ for all positive integers $\ell_{2}$ and by Lemma 4.2 , we have $S_{t}\left(P_{\frac{c}{2}+1} \cup Q_{\frac{c}{2}-1} ; \alpha\right) \leq S_{t}\left(P_{\frac{c}{2}} \cup Q_{\frac{c}{2}} ; \alpha\right)$ for all positive integers $t$. Thus $\left|A_{21}(\omega, \ell)\right| \leq\left|A_{11}(\omega, \ell)\right|$. Note that this inequality is strict for some positive integer $\ell_{0}=t_{0}+c-1$ where $t_{0} \geq \frac{c}{2}$. Also $\left|A_{21}\right| \leq\left|A_{11}\right|$ for all positive integers $\ell$, and it is strict for some positive integer $\ell_{0}$.

By similar argument as above, we can prove that $\left|A_{22}\right| \leq\left|A_{12}\right|$. Thus $\left|A_{2}\right| \leq\left|A_{1}\right|$ for all positive integers $\ell$, and it is strict for some positive integer $\ell_{0}$.

Corollary 4.1. For graphs $G(1)$ and $G(2)$ we have $E E(G(1))>E E(G(2))$. 
Proof. From Theorem 4.2, we have

$$
E E(G(2))=\sum_{\ell \geq 0} \frac{S_{\ell}(G(2))}{(\ell) !}<\sum_{\ell \geq 0} \frac{S_{\ell}(G(1))}{(\ell) !}=E E(G(1)) .
$$

The transformation from $G(1)$ to $G(2)$, depicted in Figure 3, is called transformation slowromancapi@ of $G(1)$.

Corollary 4.2. For two graphs $G^{\prime}(1)$ and $G^{\prime}(2)$, we have $E E\left(G^{\prime}(1)\right)>E E\left(G^{\prime}(2)\right)$.

Proof. By Theorem 4.1, we have

$$
E E\left(G^{\prime}(2)\right)=\sum_{\ell \geq 0} \frac{S_{\ell}\left(G^{\prime}(2)\right)}{(\ell) !}<\sum_{\ell \geq 0} \frac{S_{\ell}\left(G^{\prime}(1)\right)}{(\ell) !}=E E\left(G^{\prime}(1)\right) .
$$

The transformation from $G^{\prime}(1)$ to $G^{\prime}(2)$, depicted in Figure 2, is called transformation slowromancapi@ of $G^{\prime}(1)$. Transformation slowromancapiii@ is similar to transformation slowromancapii@ which obtained by attaching $\alpha \in G$ at $v_{0}$. There is a closed walks in $M_{c}((c-1), 0)$ which is not including the edge $v_{c} v_{c+1}$. So there is a closed walk in $M_{c}((c-1), 1)$ not in $M_{c}((c-1), 0)$. Hence, transformation slowromancapiii@ strictly decreases the Estrada index for $\ell \geq c$.

Let $G$ be a point attaching strict $k$-quasi tree graph with $k$ even cycles of length $c$, obtained by attaching the subgraphs $G_{1}, G_{2}, \ldots, G_{\frac{\Delta}{2}}$ at $u$ with the maximum degree $\Delta$. By using transformations slowromancapi@, slowromancapii@ and slowromancapiii@, $G_{i}$ s, $\left(1 \leq i \leq \frac{\Delta}{2}\right)$ can be changed into the graphs $\Gamma_{i}$ s. These transformations change $G$ into $G^{*}$ which is obtained by attaching $\Gamma_{i}$ s at $u$. Each application of transformation strictly decreases its Estrada index. So we have $E E\left(G^{*}\right)<E E(G)$. Finally repeatedly applying transformation $I, G^{*}$ can be changed into the graph $\Gamma(k)$ that is obtained from $\bigcup_{i=1}^{\frac{\Delta}{2}} \Gamma\left(k_{i}\right)$. So we have the following result.

Theorem 4.3. Let $G$ be a point attaching strict $k$-quasi tree graph with $k$ even cycles. Then $E E(\Gamma(k)) \leq E E(G)$.

\section{REFERENCES}

[1] N. Biggs, Algebraic Graph Theory, Cambridge University Press, Cambridge, 1993.

[2] D. M. Cvetković, M. Doob and H. Sachs, Spectra of Graphs: Theory and Application, Academic Press, New York, 1980.

[3] H. Deng, A proof of a conjecture on the estrada index, MATCH Commun. Math. Comput. Chem. 62 (2009), 599-606.

[4] E. Deutsch and S. Klavzar, Computing the hosoya polynomial of graphs from primary subgraphs, MATCH Commun. Math. Comput. Chem. 70 (2013), 627-644.

[5] Z. Du and B. Zhou, The estrada index of unicyclic graphs, Linear Algebra Appl. 436 (2012), 3149-3159.

[6] E. Estrada, Characterization of 3d molecular structure, Chemical Physics Letters 319 (2000), $713-718$.

[7] E. Estrada, Characterization of the folding degree of proteins, Bioinformatics 18 (2002), 697-704.

[8] E. Estrada and J. A. Rodriguez-Velazquez, Subgraph centrality in complex networks, Phys. Rev. E 71 (2005), Paper ID 056103. 
[9] I. Gutman and A. Graovac, Estrada index of cycles and paths, Chemical Physics Letters 436 (2007), 294-296.

[10] A. Ilić and D. Stevanović, The estrada index of chemical trees, J. Math. Chem. 47(1) (2010), 305-314.

[11] F. Li, L. Wei, J. Cao, F. Hu and H. Zhao, On the maximum estrada index of 3-uniform linear hypertrees, Scientific World Journal (2014), DOI 10.1155/2014/637865.

[12] F. Li, L. Wei, H. Zhao, F. Hu and X. Ma, On the estrada index of cactus graphs, Discrete Appl. Math. 203 (2016), 94-105.

[13] J. Li, X. Li and L. Wang, The minimal estrada index of trees with two maximum degree vertices, MATCH Commun. Math. Comput. Chem 64 (2010), 799-810.

[14] M. Lu and J. Gao, On the randić index of quasi-tree graphs, J. Math. Chem. 42 (2007), 297-310.

[15] Y. Shang, Biased edge failure in scale-free networks based on natural connectivity, Indian Journal of Physics 86 (2012), 485-488.

[16] Y. Shang, Random lifts of graphs: network robustness based on the estrada index, Appl. Math. E-Notes 12 (2012), 53-61.

[17] R. P. Stanley, Log-concave and unimodal sequences in algebra, combinatorics, and geometry, Annals of the New York Academy of Sciences 576 (1989), 500-535.

[18] K. Xu, J. Wang and H. Liu, The harary index of ordinary and generalized quasi-tree graphs, J. Appl. Math. Comput. 45 (2014), 365-374.

[19] J. Zhang, B. Zhou and J. Li, On estrada index of trees, Linear Algebra Appl. 434 (2011), $215-223$.

${ }^{1}$ Department of Mathematics,

YAZD UNIVERSITY

Email address: iranmanesh@yazd.ac.ir

${ }^{2}$ Department of MAthematics,

YAZD UNIVERSITY

Email address: nejati.razieh@gmail.com 